BNL - 66236

CAP-258-Muon-99

\title{
A Cupronickel Rotating Band Pion Production Target for Muon Colliders
}

\author{
Bruce J. King and Robert $\mathrm{J}$. Weggel \\ Brookhaven National Laboratory \\ and \\ Nikolai V. Mokhov \\ Fermilab \\ and \\ S. Scott Moser \\ Saint Joseph's College, Indiana \\ January 1999
}




\title{
A CUPRONICKEL ROTATING BAND PION PRODUCTION TARGET FOR MUON COLLIDERS ${ }^{1}$
}

\author{
B. J. King ${ }^{2}$, S.S. Moser $^{3}$, R.J. Weggel, BNL ${ }^{4}$; N.V. Mokhov, Fermilab
}

\section{Abstract}

A conceptual design is presented for a high power cupronickel pion production target. It forms a circular band in a horizontal plane with approximate dimensions of: 2.5 meters radius, $6 \mathrm{~cm}$ high and $0.6 \mathrm{~cm}$ thick. The target is continuously rotated at $3 \mathrm{~m} / \mathrm{s}$ to carry heat away from the production region to a water cooling channel. Bunches of $16 \mathrm{GeV}$ protons with total energies of $270 \mathrm{~kJ}$ and repetition rates of $15 \mathrm{~Hz}$ are incident tangentially to arc of the target along the symmetry axis of a 20 Tesla solenoidal magnetic capture channel. The mechanical layout and cooling setup are described. Results are presented from realistic MARS Monte Carlo computer simulations of the pion yield and energy deposition in the target. ANSYS finite element calculations are beginning to give predictions for the resultant shock heating stresses.

\section{INTRODUCTION}

High power pion production targets are required in current scenarios [1] for muon colliders. The pion secondaries from protons on the target are captured in a solenoidal magnetic channel and decay into the muon bunches needed for cooling, acceleration and injection into the collider ring. Bunched proton beams of several megawatts will be needed for the currently specified muon currents [1]: approximately $6 \times 10^{20}$ muons of each sign at repetition rates of 15 Hertz and in bunches of up to $4 \times 10^{12}$ muons per bunch. This is an extrapolation from today's high power targets in rate of target heating, shock stresses and integrated radiation damage to the target.

Because of the high beam power, liquid metal jet targets have been the subject of much recent study and form the bulk of a proposed experimental R \& D program of targetry studies that has recently been submitted [2] to the BNL AGS Division. More conventional solid targets have several challenges. Along with concerns about shock heating stresses and radiation damage, it is challenging to design a cooling scenario consistent with both the large beam power and the small target cross sections that are needed

'This work was performed under the auspices of the U.S. Department of Energy under contract no. DE-ACO2-98CH10886.

-Submutung author. email: bking@bnl.gov.

'on academic leave from Saint Joseph's College. Indiana.

${ }^{4}$ Brookhaven Vational Laboratory. P.O. Box 5000. Upton. NY 11973 $5(1000$

Fermu Nanonal Acceierator Laboratory, PO. Box 500. Batavia. IL $(0) \leq 10-0 \leq 00$

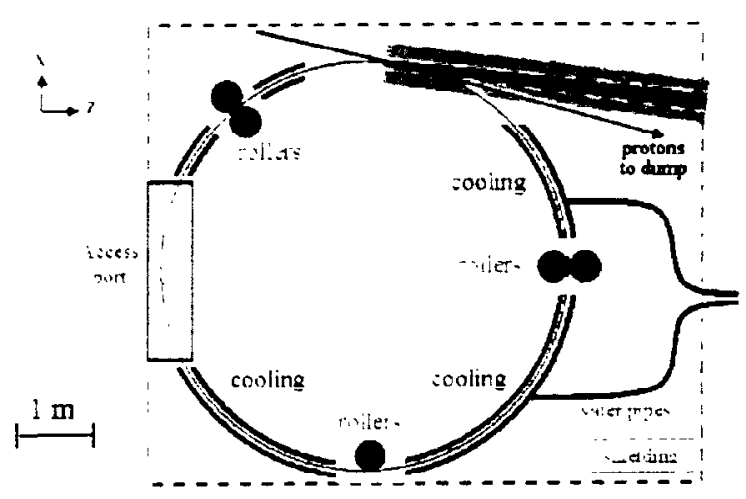

Figure 1: A conceptual illustration of the targetry setup.

for high pion yields.

This paper introduces the idea of a solid target in the form of a band that addresses this cooling issue by rotating the band to carry heat away from the targetry region and through a cooling channel.

Figures 1 and 2 give schematic views of the targetry setup we are considering and figure 3 shows the trajectory of the proton beam into the target band. It must be emphasized that details such as the rollers and cooling setup are only shown schematically and no effort has been put into their design.

The target band is enclosed in a 20 Tesla solenoidal magnetic pion capture magnet whose general design has previously been studied [1] by the Muon Collider Collaboration (MCC). The major design modification specific to this particular geometry concerns the provision of entry and exit ports for the target band.

The high-power bunched proton beam strikes the target band at a glancing angle and travels along inside the target material for two nuclear interaction lengths before the curvature of the band brings it again to an exit point at the outer edge of the band. The beam is tilted at 150 milliradians to the longitudinal axis of the solenoidal magnet; MARS simulations described below show that this gives a larger pion yield than a beam parallel to the solenoid.

\section{MARS SIMULATIONS OF TARGET HEATING AND PION YIELD}

Full MARS [3] tracking and showering Monte Carlo simulations were conducted for a $16 \mathrm{GeV}$ proton beam of 


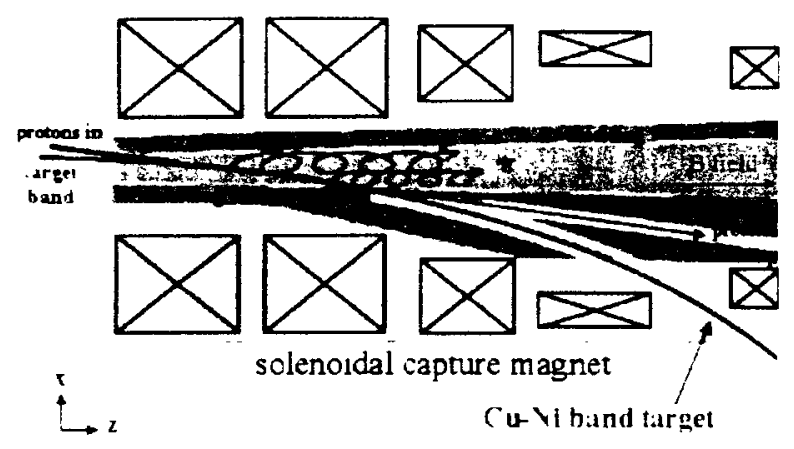

Figure 2: A conceptual illustration of the target layout around the pion production region.

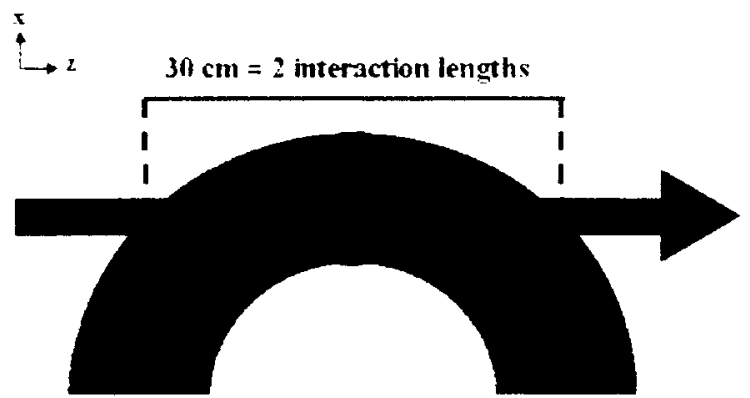

Figure 3: The trajectory of the proton beam into the target band.

$1 \times 10^{14}$ ppp with a repetition rate of $15 \mathrm{~Hz}$ on $\mathrm{Ni}$ band ( $R=250 \mathrm{~cm}, 6 \mathrm{~cm}$ height, $0.6 \mathrm{~cm}$ thickness) in a $20 \mathrm{~T}$ solenoid of $R_{a}=7.5 \mathrm{~cm}$ half-aperture. Both untilted targets and targets tilted by $\alpha=150 \mathrm{mrad}$ were studied and detailed 3-dimensional maps of energy deposition densities were generated for input to the ANSYS stress analyses.

The yield per proton at $90 \mathrm{~cm}$ downstream from the central intersection of the beam with the target was determined for pions plus muons in the momentum range $0.05<\mathrm{p}<0.8 \mathrm{GeV} / \mathrm{c}$. The yields of positive and negative pions were, respectively, $Y_{-}=0.49 \mathrm{I}$ and $Y_{-}=0.498$ at $\alpha=0$ and $Y_{-}=0.622$ and $Y_{-}=0.612$ at $\alpha=150 \mathrm{mrad}$. Figure 5 shows the momentum spectra for all hadrons and figure 6 gives more detailed information for the pions. Figure 7 shows the time distribution for when these pions are formed and rigure 8 shows several scaner plots to illustrate their distribution in phase space. These pion yields and densities in phase space are comparably good to the predictions for the best of the liquid jet targets under consideration.

The peak energy deposition density was found to be $68.6 \mathrm{~J} / \mathrm{g}$ per pulse, corresponding to a temperature rise of $\Delta T=150.5^{\circ} \mathrm{C}$. This corresponded to a total power dis- sipation in the target of $0.324 \mathrm{MW}$ at $\alpha=150 \mathrm{mrad}$. Contributions to the deposited energy come from $\mathrm{dE} / \mathrm{dx}$ from hadrons and muons $(4 \%)$, electromagnetic showering ( $46 \%)$ and from absorbtion of sub-threshold particles $(10 \%)$. Power dissipation in inner layer of tungsten shielding $(7.5<\mathrm{r}<15 \mathrm{~cm})$ was also determined. and was found to be $0.624 \mathrm{MW}$ at $\alpha=0$ and $0.766 \mathrm{MW}$ at $\alpha=150 \mathrm{mrad}$.

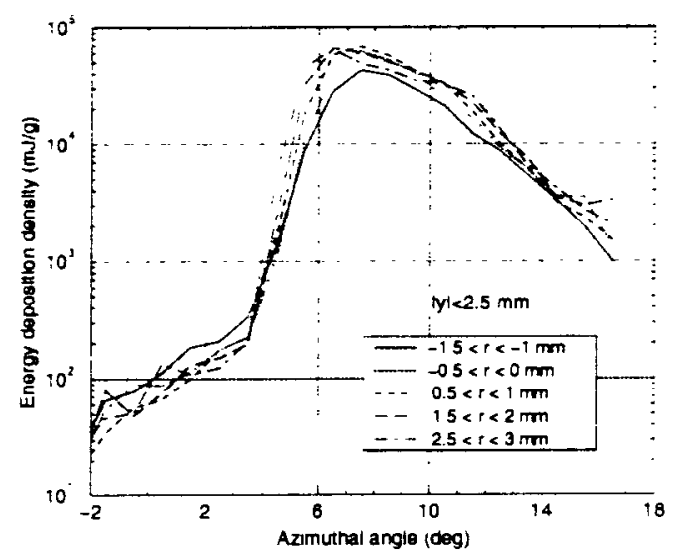

Figure 4: Energy deposition density in the band target versus azimuthal angle for a tilt angle $\alpha=150 \mathrm{mrad}$.

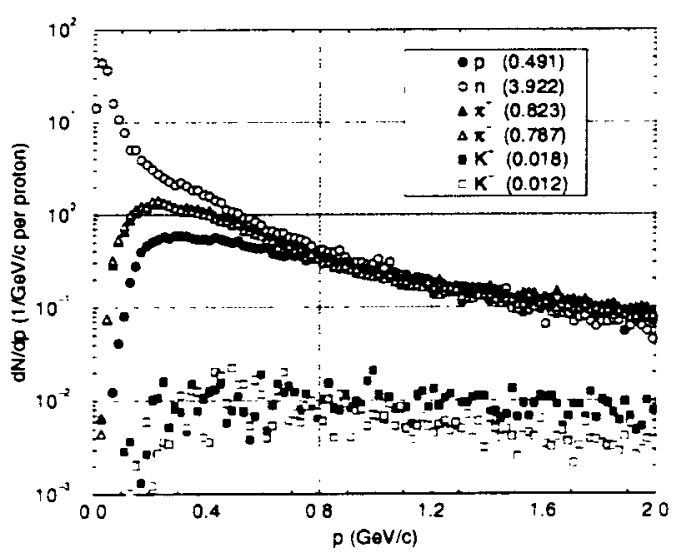

Figure 5: Momentum spectra of hadrons at $\mathrm{L}=90 \mathrm{~cm}$ and $\mathrm{R}<7.5 \mathrm{~cm}$ for a tilt angle $\alpha=150 \mathrm{mrad}$. Integrated yield is shown in parentheses.

\section{ANSYS STRESS SIMULATIONS}

The survivability of solid targets in the face of repeated shock heating is probably the most challenging problem faced in these scenarios for pion production for muon colliders.

To investigate this, we are beginning to conduct finite element computer simulations of the shock heating stresses 


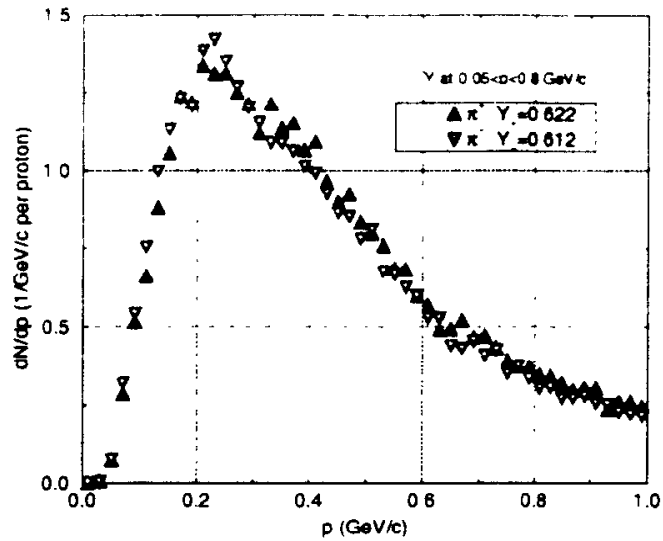

Figure 6: Pion momentum spectra at $\mathrm{L}=90 \mathrm{~cm}$ and $\mathrm{R}<7.5 \mathrm{~cm}$ for a tilt angle $\alpha=150 \mathrm{mrad}$.

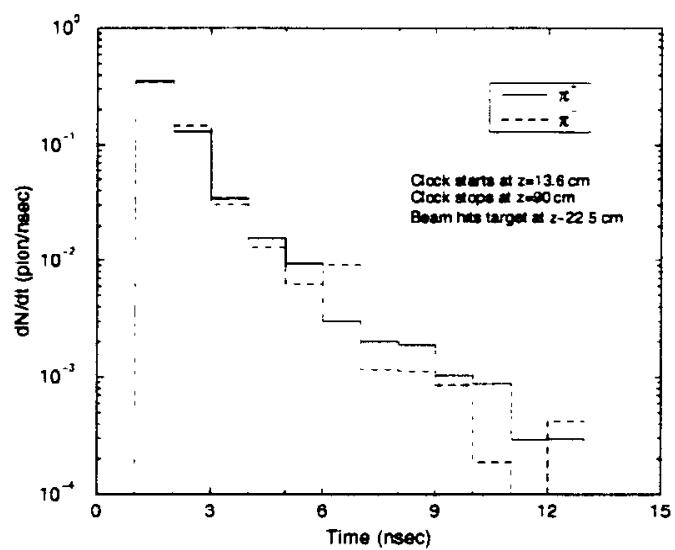

Figure 7: Pion time spectra at $\mathrm{L}=90 \mathrm{~cm}$ and $\mathrm{R}<7.5 \mathrm{~cm}$ for $0.05<\mathrm{p}<0.8 \mathrm{GeV} / \mathrm{c}$ and a tilt angle $\alpha=150 \mathrm{mrad}$.

using ANSYS, a commercial package that is very widely used for stress and thermal calculations. These studies are still at an early stage.

It is encouraging that the instantaneous temperature rise predicted by MARS of approximately 70 degrees per proton pulse is much less than the 500-600 degree temperature rise in microsecond timescales that the Fermilab pbar source nickel target routinely operates at. Further, if the predicted stresses turn out to be higher than, say, $50 \%$ of the target's tensile strength then possibilities exist for redimensioning the target and the proton spot size to reduce the stress.

\section{CONCLUSIONS}

In conclusion, initial studies indicate that cupronickel rotating band targets may well be a viable and attractive option to satisfy the difficult high power targetry requirements of

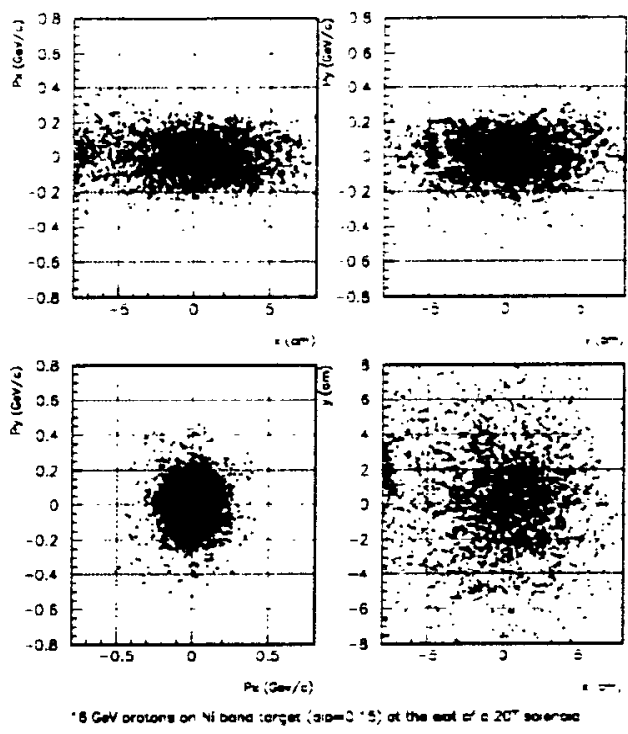

Figure 8: $\pi^{-}$(red) and $\pi-$ (green) scatter plots at $L=90 \mathrm{~cm}$ and $R<7.5 \mathrm{~cm}$ for a tilt angle $\alpha=150 \mathrm{mrad}$.

muon colliders.

\section{REFERENCES}

[1] The Muon Collider Collaboration. "Status of Muon Collider Research and Development and Future Plans". to be submitted to Phys. Rev. E.

[2] Alessi et al., "An R \& D Program for Targetry and Capture at a Muon-Collider Source - A Proposal to the BNL AGS Division". Spokesperson Kirk T. McDonald email: mcdonald@puphep.princeton.edu .

[3] N.V Mokhov, "The MARS Code System User's Guide. Version 13 (98)", FERMILAB-FN-628 (Feb. 1998). 